\title{
Spectrum Sensing in Cognitive Radio using Frequency Domain
}

Sasikar. A

\begin{abstract}
An efficient bandwidth allocation and dynamic bandwidth access away from its previous limits is referred as cognitive radio (CR).The limited spectrum with inefficient usage requires the advances of dynamic spectrum access approach, where the secondary users are authorized to utilize the unused temporary licensed spectrum. For this reason it is essential to analyze the absence/presence of primary users for spectrum usage. So spectrum sensing is the main requirement and developed to sense the absence/ presence of a licensed user. This paper shows the design model of energy detection based spectrum sensing in frequency domain utilizing Binary Symmetric Channel (BSC),Additive white real Gaussian channel (AWGN), Rayleigh fading channel users for 16-Quadrature Amplitude Modulation(QAM) which is utilized for the wide band sensing applications at low Signal to noise Ratio(SNR) level to reduce the false error identification. The spectrum sensing techniques has least computational complexity. Simulink model for the energy detection based spectrum sensing using frequency domain in MATLAB 2014a.
\end{abstract}

Keywords : CR, Simulink, Energy detection, spectrum sensing.

\section{INTRODUCTION}

For past few years cognitive radio research has established as a considerable interest in communication field. Fig.1 describes the spectrum sensing based CR. Basically the spectrum sensing systems are categorized into following type blind based and knowledge based methods.

Nonparametric cyclic correlation estimator is described in [1]. For minimizing the time of a sequential detection approach is used. This method is realized in non-Gaussian and Gaussian noise situation. To reduce the noise effect multi slot cyclostationary feature detector is described in [2]. Overflow/under flow protection for time domain cyclostationary feature detector is described in [3]. This method supports overall range of the subcarriers utilized by OFDM to 4G LTE. To enhance the spectrum sensing performance cascaded filter bank channelization is described in [4].This method supports offers less power. To reduce power in CR network sensing time period is described in [5] by using squaring law with decision rule. Model using Welch periodogram and FFT is designed in [6].In this method

Revised Manuscript Received on December 30, 2019.

* Correspondence Author

Sasikar A*, Assistant Professor, Department of Electronics and Communication Engineering,Vel Tech Rangarajan Dr.Sagunthala R\&D Institute of Science and Technology, Avadi, Chennai, India. Email: sasikara@veltech.edu.in.

(c) The Authors. Published by Blue Eyes Intelligence Engineering and Sciences Publication (BEIESP). This is an open access article under the CC BY-NC-ND license (http://creativecommons.org/licenses/by-nc-nd/4.0/)
Neyman Pearson hypothesis is considered. In [7], Neyman Pearson is used for energy detection method and for validating such algorithm various modulated signals that is BPSK, QPSK, DVB-T under Rayleigh fading and white

Gaussian noise is described in [9] and [10]. This method provides less false detection. Low Power and area efficient SRAM structure on the basis of the GDI is designed in [12]. Smart Energy Tracking System on IOT is defined in [13]. In [14], wireless sensor applications for lively communication predictive re-alignment approach is described. For cancelling noise, in [15] they used the cascaded combination of adaptive and wavelet filter.

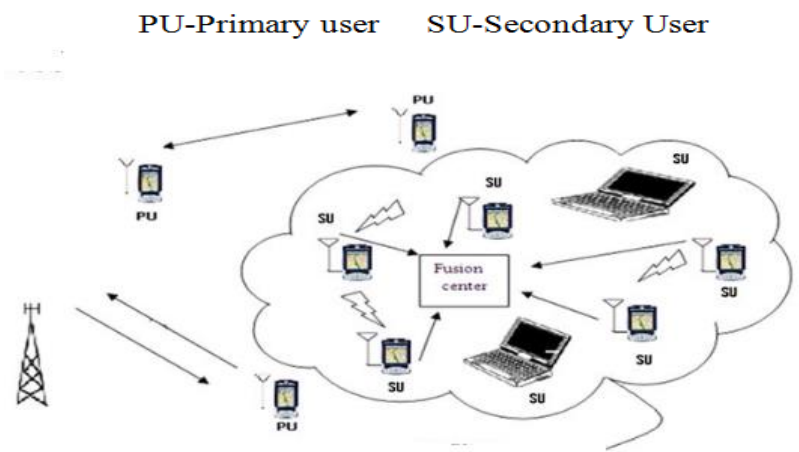

Fig. 1.Spectrum sensing in CR network.

The previous researchers mainly focus on the time domain based energy detector which increases the false detection at low SNR [16]. To overcome this problem the proposed method designed the energy detector model with less false detection of users in CR applications. Further this manuscript is planned as follows: related works in section 2, proposed works in section 3, simulation result in section 4 and Conclusion in section 5 .

\section{RELATED WORK}

In conventional method [6] the energy detector made up of low pass filter to avoid the adjacent signals and band noise, integrator, square law device and Analog to Digital Converter (ADC) is realized in time domain which is shown the Fig.2. But for sine waves and narrow band signals such type of realizations is hard. To overcome this problem the proposed method uses squaring the FFT magnitude to calculate the spectrum in CR network. 


\section{Spectrum Sensing in Cognitive Radio using Frequency Domain}

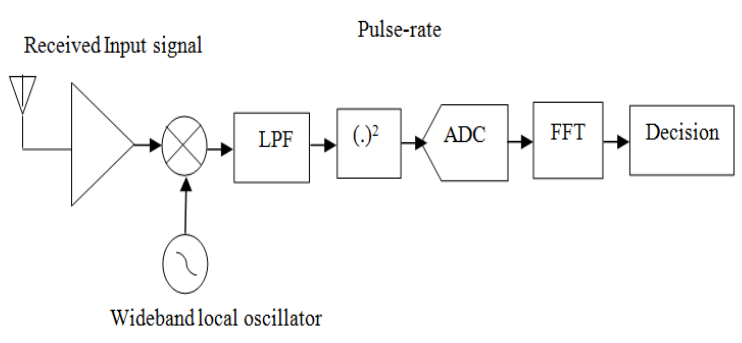

Fig. 2.Conventional energy detection based spectrum sensing techniques.

\section{PROPOSED METHOD}

If The digital signal energy over $\mathrm{N}$ samples can be computed by,

$$
E=\sum_{n=0}^{N-1}|I(n)|^{2}
$$

Where I(n) is digital signal energy. The above equation used for the time domain. If the received signal is degraded by noise, there is the chance to mislead between the noise and signal in time domain detector. To overcome this problem the frequency domain energy detector is used to sense the primary user presence at low SNR with fewer false alarms compare to the conventional method. Frequency domain makes use of power spectral density(PSD) of received primary users. To compute the PSD the time domain signal $\mathrm{I}(\mathrm{n})$ is converted to frequency domain.

$$
\begin{gathered}
I(k)=\frac{1}{N} \sum_{n=0}^{M-1} I(n) \cdot e^{\left(\frac{-j 2 \pi k n}{N}\right)} \\
P(k)=\sum_{n=0}^{N-1}|I(k)|^{2} \\
I(\omega)=\sum_{n=-\infty}^{\infty} I(n) \\
I(n)=\frac{1}{N} \int_{\omega=-\pi}^{\pi} x(\omega) e j{ }^{\omega n} d w
\end{gathered}
$$

By considering various channels such as BSC, AWGN and Rayeleigh fading channel the proposed method developed the energy detection based spectrum sensing. At a very regular case the AWGN is available where the noise is primarily identified by the front-end analog receiver thermal noise. The transmitted signal is equal to the received signal and noise which gives the equality in communication applications. BSC is used in coding theory and information theory which transmits and receives the binary bits. Rayleigh fading noise signal is passed through the communication channel by assuming the magnitude in the signal. The design procedure for the proposed model is:

i) Through ADC the received signal is passed and next the coefficient of FFT is calculated.

ii) Using the observation interval the above coefficient values are squared and averaged.

iii) To fix the primary user presence, the result values of edge detector is evaluate with predefined decision threshold value utilizing binary symmetric channel and Additive Gaussian white noise.
Fig.3 shows the proposed energy detector model utilizing FFT. In this model first through band pass filter the analog signal is filtered and converted into the digital signal. A 16-bit ADC quantizer with $=5 \mathrm{~V}$ and $=0 \mathrm{~V}$ has been utilized. To realize the corresponding filter coefficients the output of the ADC is passed to the FFT module. Using such module the time is transformed into the frequency domain. The received signal magnitude is squared and the less amount of wave is considered to be noise. If the threshold range is exceeded the received wave is present with the help of the relational operator such process is carried out.

\section{SIMULATION RESULTS}

The energy detector model using frequency domain for 16-QAM is simulated using MATLAB 2014a Simulink. Where the primary users are generated and the signal is summed with the estimation and noise is taken utilizing FFT. To find the band engaged by the PU the threshold is computed using the relational operator. Here the noise channels are AWGN, BSC, and Rayleigh fading channel. Fig. 4 shows FFT spectrum estimation. At point 2 defines the threshold level, here the above level of threshold are the occurrence of primary user whereas below the threshold level are secondary users.

In conventional method QAM is found to be better so here QAM model is utilized. Generally QAM is made up of quadrature phase and in-phase components which vary in amplitude and phase. The energy detection of the received signal is considered to be QAM.SNR is the element to be considered in the method. Table I shows the performance result for 16-QAM energy detector using frequency domain for different channel users.

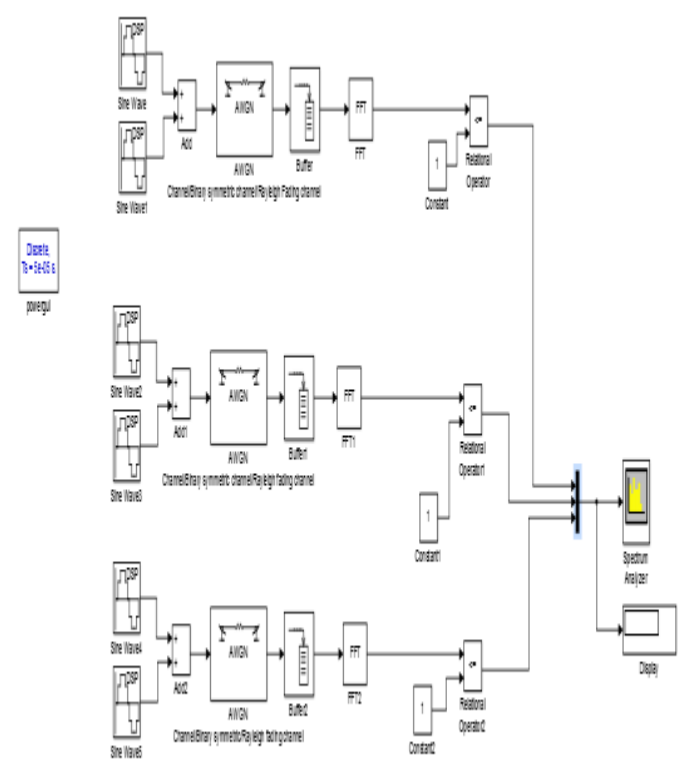

Fig. 3.Simulink model for the energy detection using frequency domain.

Published By:

Blue Eyes Intelligence Engineering \& Sciences Publication 


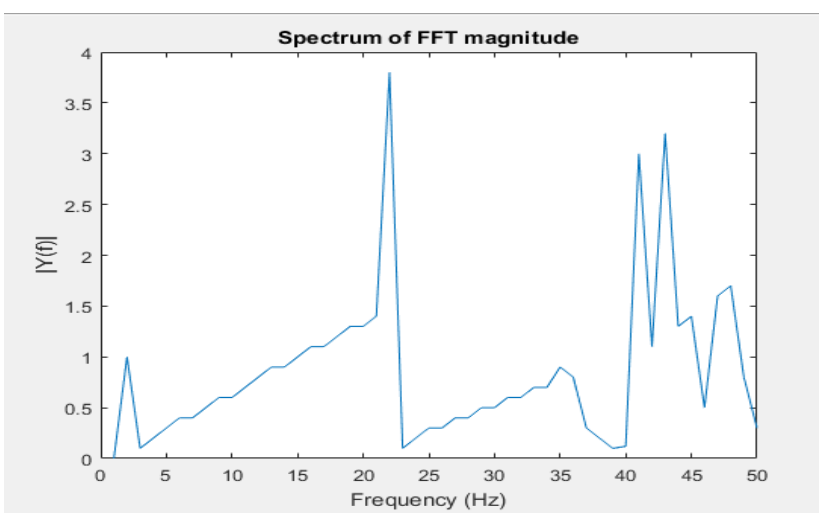

Fig. 4.Spectrum estimation of FFT block .

From this table the AWGN channel user has less false detection at low SNR level compare to the BSC channel \& Rayleigh fading channel users.

Table- I: Performance result of the energy detector for 16-QAM at $-1 \mathrm{~dB}$ (SNR)

\begin{tabular}{|c|c|c|}
\hline Channel & $\begin{array}{c}\text { Probability of } \\
\text { detection }\end{array}$ & $\begin{array}{c}\text { Probability of } \\
\text { Misdetection }\end{array}$ \\
\hline $\begin{array}{c}\text { AWGN } \\
\text { channel }\end{array}$ & 1.0012 & 0 \\
\hline $\begin{array}{c}\text { BSC } \\
\text { channel }\end{array}$ & 1.0114 & 0.003 \\
\hline $\begin{array}{c}\text { Rayleigh } \\
\text { fading } \\
\text { channel }\end{array}$ & 1.0220 & 0.001 \\
\hline
\end{tabular}

\section{CONCLUSION}

In this paper the spectrum sensing using frequency domain are discussed for the CR network applications. In such application the probability of detection raises as the SNR raises thereby minimizing the probability of misdetection. Here, Simulink model for energy detection using frequency domain with AWGN, binary symmetric and Rayleigh fading channel are used for the users. From the comparison table the probability misdetection and probability detection for 16-QAM , the AWGN user has less false detection compare to the other channel user at low level SNR .The AWGN channel offers zero probability of misdetection at the $-1 \mathrm{~dB}$ SNR level range.

\section{REFERENCES}

1. J. Lundén, S. A. Kassam, and V. Koivunen, "Robust nonparametric cyclic correlation-based spectrum sensing for cognitive radio", IEEE Transactions on Signal Processing, 58(1),2009, pp.38-52.

2. I. G. Anyim, J. Chiverton, M. Filip, and A. Tawfik. "The optimization of wideband cyclostationary feature detector with receiver constraints",2017,pp. 8-6.

3. M. S. Murty, and R. Shrestha," Hardware implementation and VLSI design of spectrum sensor for next-generation LTE-A cognitive-radio wireless network", IET Circuits, Devices \& Systems, 12(5),2018, pp.542-550.

4. T. H. Yu, C. H., Yang, D. Markovic, and D. Cabric, "An energy-efficient VLSI architecture for cognitive radio wideband spectrum sensing", IEEE Global Telecommunications Conference-GLOBECOM , Dec.2011,pp. 1-6.

5. M. Pirmoradian ,O. Adigun , C. Politis" Sensing optimization in cooperative cognitive radio networks", Procedia Computer Science, Vol. 1,No.34, Jan.2014,pp.577-82.

6. V. Divyapraba, K. Kishore, R. Pratheepa, and V. Elamaran, "Spectrum sensing based on energy detection using MATLAB_Simulink", Indian Journal of Science and Technology, Vol.8,No.29,Nov.2015 ,pp.1-5.
7. S. L. Srinu , S. L. Sabat ," FPGA implementation of spectrum sensing based on energy detection for cognitive radio", IEEE International Conference on Communication Control And Computing Technologies Oct.2010, pp. 126-131.

8. S. Xia, and L. Zhang, "Optimization for cooperative spectrum sensing in cognitive radio networks", IEEE International Symposium on Intelligent Information Technology Application ,Vol. 3, Nov.2009, pp. 331-334.

9. S. Pavithra,S. Karthikeyan,V. K. Sonti, and S. Jayashri, S.,'Competent realisation of cooperative spectrum sensing in cognitive radio systems", International Journal of Engineering Systems Modelling and Simulation, Vol.7,No.2,Jan.2015, pp.103-110.

10. V. N. Kumar, H. Bhalavi, G. Lakshminarayanan, and M. Sellathurai, "FPGA based decision making engine for cognitive radio using genetic algorithm", IEEE 8th International Conference on Industrial and Information Systems,Dec.2013,pp. 633-636

11. D. Teguig ,M. S. Azzaz," FPGA implementation of Spectrum Sensing methods for Cognitive Radio", IEEE International Symposium on Networks, Computers and Communications ,Jun. 2018,pp. 1-5.

12. C. Jeyasree, and R. A. Raj, “ Design Of Low Power And Area Efficient Sram Architecture Based On Gdi”, International Journal of MC Square Scientific Research Vol.9, No.1, April. 2017,pp.18-25.

13. R. Jayanthi, S. T. Rama ," IOT Based Smart Energy Tracking System”, International Journal of MC Square Scientific Research , Vol.9,No.1,2017,pp.98-108.

14. B. M. Vallapuram , G. P. Nair, "Predictive re-alignment strategy for agile communication in wireless sensor networks". International journal of advances in signal and image sciences, Vol.20,No.1,Dec.2015,pp.12-8.

15. S. Mishra,"Cascade combination of wavelet and adaptive filter for noise cancellation", International journal of advances in signal and image sciences, Vol.2,No.2,Dec.2016,pp.21-6.

16. T. A. Shanmugasundaram, and V. Vijayabaskar, "Bit Error Rate Analysis of RS (7, 3) Coded Frequency Shift Keying Using Simulink", American Journal of Applied Sciences, Vol. 12, No. 2, Mar.2015, pp. 92-98.

\section{AUTHORS PROFILE}

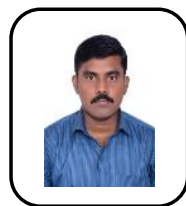

Sasikar A, received the B.E. degree in Electronics and Communication Engineering from Dr.Sivanthi Aditanar College of Engineering, Tiruchendur, Tamilnadu, India in 2004, the M.E. degree in Applied Electronics from College of Engineering Guindy (CEG), Anna University, Chennai, Tamilnadu, India, in 2011.

He is currently working as an Assistant Professor in the Department of Electronics and Communication Engineering, Vel Tech Rangarajan Dr.Sagunthala R\&D Institute of Science and Technology, Avadi, Chennai, Tamilnadu, India. His research interests include Low power VLSI design, FFT Algorithms 